\title{
Simulation of Transition Radiation and Electron Identification Ability of the ATLAS TRT
}

\author{
E. Klinkby on behalf of the ATLAS collaboration \\ Niels Bohr Institute, HEP \\ Risø-DTU, NUK
}

\section{Abstract}

The Transition Radiation Tracker (TRT) is the outermost tracking detector of the ATLAS experiment. In addition as functioning as a tracking detector, it is capable of providing particle identification information, through the emission, and subsequent absorption and detection of transition radiation. Below the effort of simulating transition radiation production and detector response is discussed, with emphasis on the data/simulation resemblance and tuning.

\section{Introduction}

Transition radiation (TR) arises when ultra-relativistic charged particles cross a boundary between media with different dielectric constants, i.e. a charged particle in a non-uniform electric field. This process is analogous to bremsstrahlung, which arises due to a particle moving in a electromagnetic field. As shown below, the probability of a given particle to emit transition radiation is determined solely by the Lorentz $\gamma$-factor (apart from material properties). The TRT is built to exploit this for particle identification purposes - in particular to distinguish electrons from pions, in the low energy region $(E<10 \mathrm{GeV})$ where the electromagnetic calorimeter loses separation power. Electrons tend to have large $\gamma$-factors and thus are likely to emit TR photons which makes them separable from the abundant low energy background of mainly pions. The theory of transition radiation is treated in detail in $[1,2]$ and the references therein. Only a few results important for the simulation of TR in the TRT are summarised below. The implementation of transition radiation simulation in the ATLAS experiment is based directly on [1], and the GEANT4 default is not used. The main reason for this is that historically the TR model in GEANT4 was poorly suited for the transition radiation simulation needed by in the TRT software since it could not take into account interference between radiation produced at different gas-foil transitions.

\section{Theory of transition radiation}

In the case of a single regular foil, the total energy radiated by a charged particle when passing from vacuum to the foil is:

$$
E_{\text {rad }}=\frac{1}{3} \alpha \gamma \hbar \omega_{p}
$$

where $\omega_{p}$ is plasma frequency of the foil and is given by:

$$
\hbar \omega_{p}=\frac{\sqrt{4 \pi N_{e} r_{e}^{3}} m_{e} c^{2}}{\alpha}
$$

where $N_{e}$ is the electron density, $r_{e}$ is the classical electron radius, $m_{e}$ is the electron mass and $\alpha$ is the fine-structure constant.

Strictly speaking, this result is only valid in case the plasma frequency of the surrounding medium, $\omega_{\text {gas }}$, is zero, which is the case for a transition from vacuum. However, the result holds approximately in the limit: $\omega_{\text {foil }} \gg \omega_{\text {gas }}$ as is the case for the relevant materials of the TRT: $\hbar \omega_{\text {polypropylene }} \approx 20 \mathrm{eV}$ and $\hbar \omega_{\text {air }}=0.70 \mathrm{eV}$. For practical usage, not all transition radiation photons are useful, since some minimum energy, $E_{\text {cutoff }}$ must be required for detection (due 
to the absorption spectrum in the detection gas). The number of photons created at a single boundary fulfilling this requirement is Poisson distributed with a mean of:

$$
N_{\gamma}=\frac{\alpha}{\pi}\left(\ln \frac{\hbar \omega_{p} \gamma}{E_{\text {cutoff }}}\left(\ln \frac{\hbar \omega_{p} \gamma}{E_{\text {cutoff }}}-2\right)+\frac{\pi^{2}}{12}+1\right)
$$

The TRT is designed to be sensitive to photons with energies above $1 \mathrm{keV}$ thus implying that an electron of $100 \mathrm{GeV}$ on the average produces $\approx 0.12$ photons with sufficient energy. For detection the low single-foil emission probability is compensated by using many foil layers. In the TRT end-cap each straw-layer is interleaved with about 15 uniform regular $15 \mu \mathrm{m}$ thick polyethylene foils separated by $200 \mu \mathrm{m}$ gas gaps (300 $\mu \mathrm{m}$ in some regions). Studies show (see figure 1(left)) that this composition is optimal (due to constructive interference of the radiation, regular foils are better than non-regular foils, foam and fibres). The thickness and number of foil layers are optimised with respect to PID performance, subject to constraints from the material budget and space limitations. The geometry and modular design of the TRT barrel prevents usage of regular foil radiators and an assessment shows that fibres oriented perpendicular to the expected particle trajectory is the best practical option given these constraints - see figure 1(left). At nominal threshold $(5-7 \mathrm{keV})$, the probability for exceeding threshold is here about $15 \%$ below that of a regular foil.
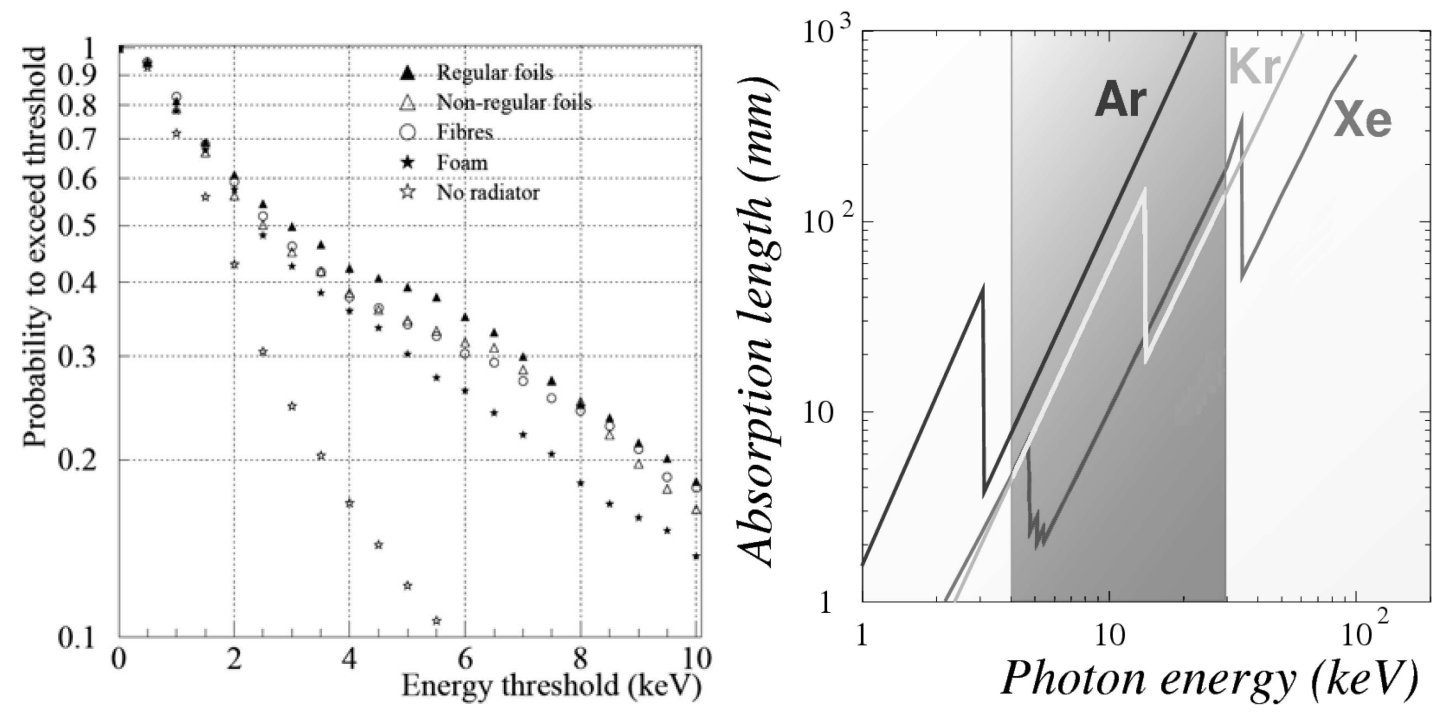

Figure 1: Left: High-threshold (HT) probabilities as a function of threshold for different radiator types. Right: Photon absorption length in high $\mathrm{Z}$ noble gases. Source [3].

Upon creation, the TR photons, which are not re-absorbed in the foils or in the intermediate gas, enter the straws of the TRT and are subsequently absorbed in the Xenon gas flushed through the straws of the TRT detector. Xenon is chosen based on its low photon absorption length at the relevant photon energies (see figure 1 (right)) while other gases are added for stability, drift velocity and detector safety reasons. The final composition is $\mathrm{XeCO}_{2} \mathrm{O}_{2}(70: 27: 3)$.

The TR photons are emitted highly collinear to the particle trajectory (opening angle $\theta \approx 1 / \gamma$ ) As a result of this collinearity, the fraction of hits along the trajectory of a given particle which contain high threshold (HT) hits can be easily interpreted as a HT probability and be used in a straight-forward manner for particle identification.

\section{Simulating transition radiation}

In this section the tuning of the TR model to match Combined Test Beam (CTB) data is discussed [4]. 


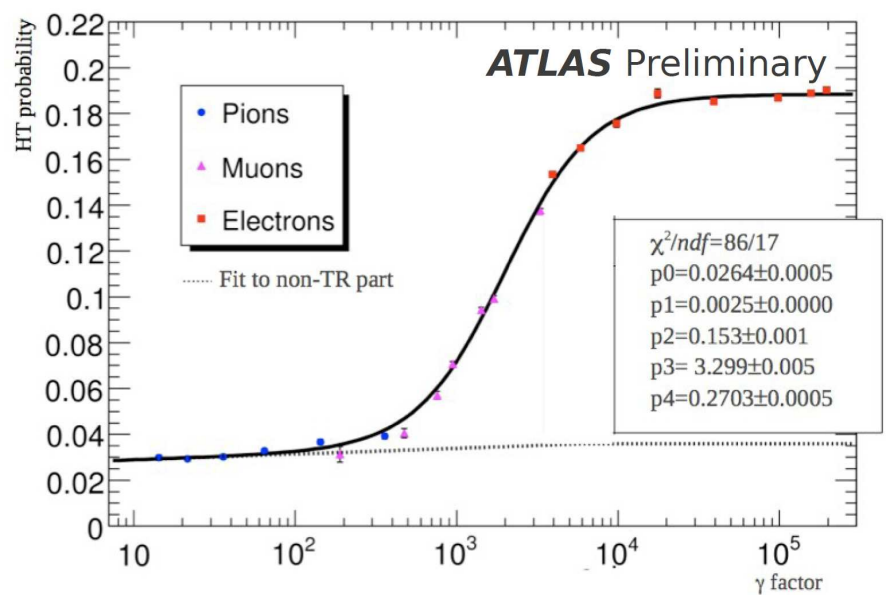

Figure 2: HT probabilities for different particles as a function of $\gamma$-factor from test-beam of barrel modules [5].

Figure 2 shows the HT probability from a study of the CTB, as a function of $\gamma$-factor. The plot is the result of a careful track selection and strict requirements ensuring reliable and high separation between the particle species. The contamination is estimated to be below $0.1 \%$ in all configurations, and in most samples considerably lower than that. As would be expected from the above considerations, the HT probability curve resulting from the selection (figure 2) shows no dependence on the particle species, only on the $\gamma$-factor - i.e. there is no discontinuity in the curve in the regions between different particle species. The function used for the fit is a generic onset function of the form:

$$
p_{H T}(\gamma)=p_{0}+p_{1} \log _{10}(\gamma)+\frac{p_{2}}{1+\exp \left(-\left(\log _{10}(\gamma)-p_{3}\right) / p_{4}\right)}
$$

The various components of this function are discussed in the next sections.

To reduce ambiguities in track-selection from : $e^{-} \rightarrow e^{-} \gamma \rightarrow e^{-} e^{+} e^{-}$and pion decays, the Monte Carlo study is based entirely on muons. The muon energies are selected in such a way that their $\gamma$-factors match those of the Combined Test Beam - i.e. a muon run corresponding to each point in figure 2.

The tuning of the transition radiation model proceeds in three steps: First the low energy $d E / d x$ tail is tuned, then the TR onset part is tuned and finally the high energy plateau.

\subsection{Step 1: Tuning the $d E / d x$ tail}

Figure 3(left) shows the default Monte Carlo HT probability corresponding to the test-beam data of figure 2. Low energy ionisation has been subject to intense study during a period of many years. Most of the important results are implemented in the Photo Absorption Ionisation (PAI) model[6] which in this domain is very trustworthy. It is therefore considered safe, to tune the low $\gamma$ tail of figure 3(left) to data using as tuning parameter the HT setting. Switching off the TR model in the simulation gives the pure $d E / d x$ curve shown in figure 4.

\subsection{Step 2: The transition radiation onset}

A study of the transition radiation model implementation in the ATLAS software reveals a potential problem for the barrel modules. The fibres responsible for creating the TR photons is in lack of a better model approximated by the same foil as for the end-cap. Differences in the transition radiation production are introduced only via a constant factor whose effect is to disregard a certain fraction of the created TR photons in the barrel with respect to the end-caps (named TR efficiency in the following).

In order to force the onset curve of the MC to match that of data, the number of created $\mathrm{MC}$ transition radiation photons is modified according to the ratio between the fits to the HT 

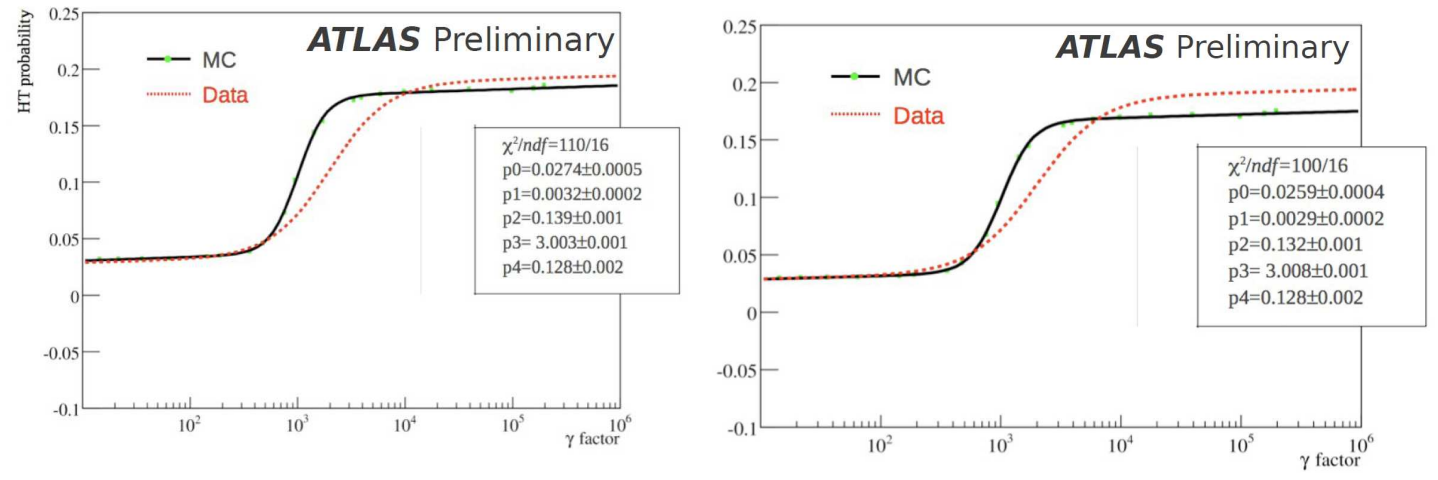

Figure 3: Tuning the Monte Carlo HT low energy plateau probabilities as a function of $\gamma$ factor. The curves are based on 21 points, each resulting from a simulation of 10000 tracks. On the left is shown the result obtained using the default setting: $\mathrm{HT}=6 \mathrm{keV}$ whereas the right-hand figure shows the result after tuning the low energy plateau to data: $\mathrm{HT}=6.25 \mathrm{keV}$. To be comparable with figure 2, the simulation is done for barrel only. Note that in both plots, the TR efficiency is set to the somewhat arbitrary default setting: 0.8 (explained below). The dotted curves shown in both plots is the data fit as described by the parameters in figure 2 [5].

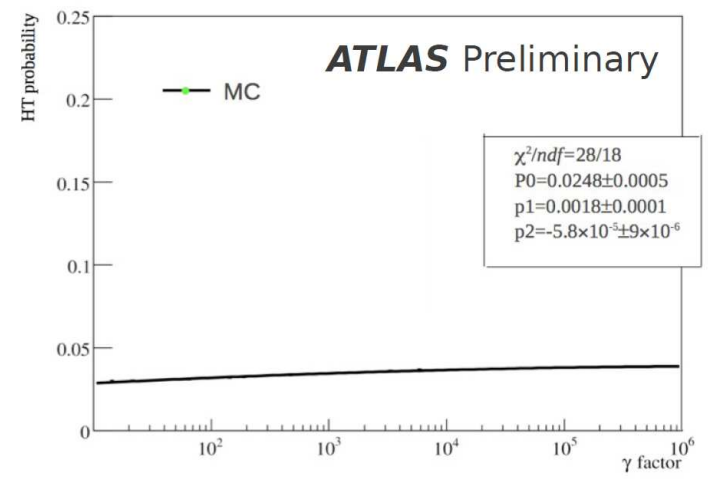

Figure 4: Left: $\mathrm{MC} d E / d x$ curve in the full range after tuning the low energy tail to data. Obtained by switching off the TR creation. Barrel only [5]. 


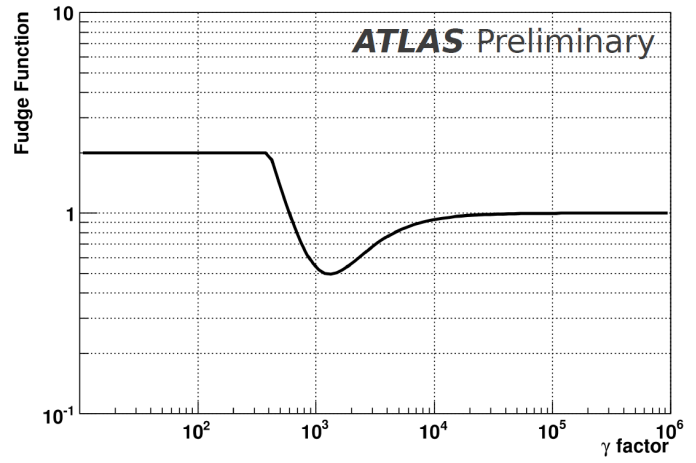

Figure 5: Fudge function scaling the number of photons in MC to give the TR onset curve of data. The maximal allowed value of the fudge function is 2 , and the function is relevant only in the TRT barrel [5].

probability curves for data and MC after subtracting the $d E / d x$ curve shown in figure 4 . The ratio of the fits of figures 2 and 3 has the functional form:

$$
N_{\gamma} \rightarrow N_{\gamma} \cdot \frac{1+e^{-(\log \gamma-3.008) / 0.1284}}{1+e^{-(\log \gamma-3.299) / 0.2703}}
$$

where $N_{\gamma}$ is a Poisson number with mean as given by equation 3 . The function is shown in figure 5 - including a cutoff, implemented so that the correction do not exceed a factor of 2 . The cutoff is needed to avoid incorrectly interpreting and artificially scaling small differences between the low energy tails of the data and MC curves. Only $d E / d x$ is relevant to the low energy tail and the tail should be scaled factor resulting from the effect of transition radiation. The reason to subtract the $d E / d x$ curve is that only the TR model is to be modified - not the $d E / d x$ part underneath which is assumed valid.

The resulting HT probability curve is shown in figure 6(left) along with the CTB curve. Clearly, the main features of the TR onset are now effectively reproduced in the simulation, the only remaining issue is to tune the high energy plateau which is done below.

\subsection{Step 3: The saturation level}

Since the number of transitions between gas and fibres is finite and since some fraction of the TR photons are re-absorbed in the various materials, the HT probability saturates at a certain $\gamma$-factor. The final step in the tuning concerns the simple matching of the saturation plateau in data and MC. In the simulation (actually in the digitization) this is done by tuning the overall TR efficiency. The final result of the tuning is shown in figure 6(right). The corresponding TR efficiency is $95 \%$.

\section{Conclusion}

The method and results described in this paper are implemented in the ATLAS simulation software [7]. In the present situation, where the HT probability curve of MC matches data well, it is clear that PID information can be extracted in data as well as in reconstructed MC solely based on the HT probability curve. While not shown in this paper, an additional approach is also developed where not only HT probability, but also signal time-over-threshold are used to form a single PID probability. For a detailed discussion on the PID performence of both the highthreshold based and the time-over-threshold based approach the reader is referred to reference: $[8]$.

[1] B. Dolgoshein, Transition Radiation Detectors and Particle Identification, Nucl. Instrum. Meth. A252 (1986) 137-144. doi:10.1016/0168-9002(86)91174-5. 

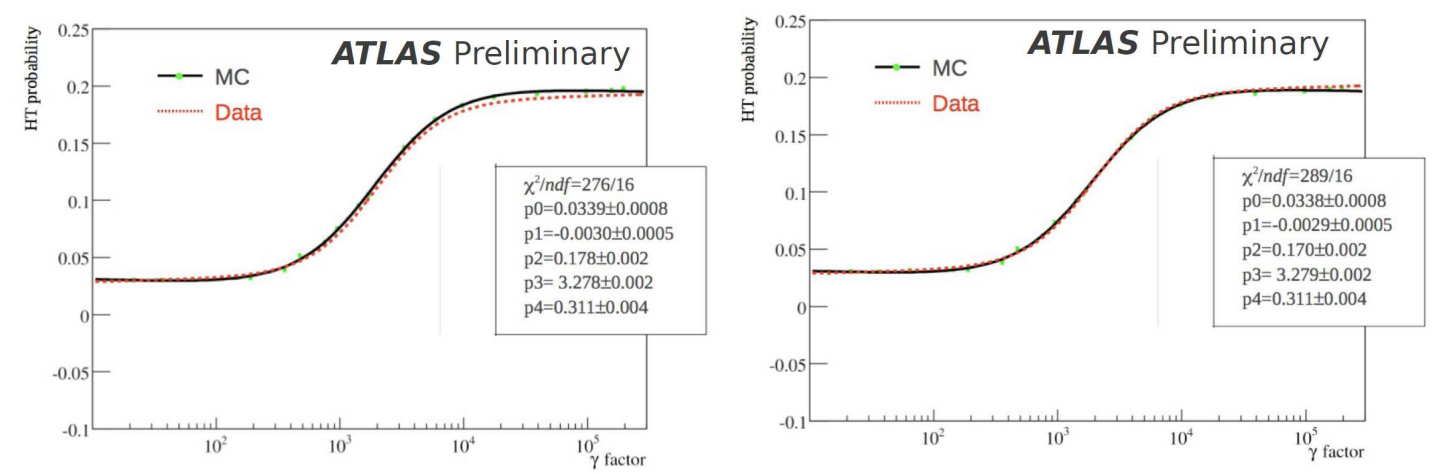

Figure 6: Modified MC (full curve) and data (dotted curve). Left: TR efficiency 100\%, Right: TR efficiency $95 \%$. Both plots are for barrel only [5].

[2] U. Egede, The search for a Standard Model Higgs at the LHC and electron identification using transition radiation in the ATLAS tracker, CERN-THESIS-98-001.

[3] P. Cwetanski, Straw performance studies and quality assurance for the ATLAS transition radiation tracker, CERN-THESIS-2006-025.

[4] E. Abat, J. Abdallah, T. Addy, P. Adragna, M. Aharrouche, et al., Combined performance studies for electrons at the 2004 ATLAS combined test-beam, JINST 5 (2010) P11006. doi:10.1088/1748-0221/5/11/P11006.

[5] https://twiki.cern.ch/twiki/bin/view/AtlasPublic/TRTPublicResults.

[6] W. W. M. Allison, J. H. Cobb, Relativistic Charged Particle Identification by Energy Loss, Ann. Rev. Nucl. Part. Sci. 30 (1980) 253-298.

[7] E. B. Klinkby, W mass measurement and simulation of the transition radiation tracker at the ATLAS experiment, CERN-THESIS-2008-071.

[8] ATLAS Collaboration, Particle Identification Performance of the ATLAS Transition Radiation Tracker, ATLAS note ATLAS-CONF-2011-128, https ://cdsweb.cern.ch/record/1383793. 